\title{
ANÁLISE DA VIABILIDADE TÉCNICA E ECONÔMICA DE ESTRUTURA DE ELEVAÇÃO PARA UM SISTEMA FOTOVOLTAICO EM UM PRÉDIO DE UMA UNIVERSIDADE
}

\author{
Alisson Felipe Urbano de Almeida (1); Andréa Teresa Riccio Barbosa (2)
}

(1) Engenheiro Eletricista, mestrando no Programa de Pós-Graduação Stricto Sensu em Eficiência Energética e Sustentabilidade - Curso de Mestrado Profissional da Universidade Federal do Mato Grosso do Sul, alissonurbano1995@gmail.com, Campo Grande, MS, Tel.: (67) 99979-9036.

(2) Engenheira Eletricista, Dra., Professora do Programa de Pós-Graduação Stricto Sensu em Eficiência Energética e Sustentabilidade - Curso de Mestrado Profissional da Universidade Federal do Mato Grosso do Sul, andrea.barbosa@ufms.br, Universidade Federal de Mato Grosso do Sul, FAENG - Faculdade de Engenharias, Arquitetura e Urbanismo e Geografia, Campo Grande - MS, Caixa Postal 549, CEP 79070-900, Tel.: (67) 3345-7475.

\section{RESUMO}

A energia elétrica tem se tornado cada vez mais importante na sociedade ao passar dos anos. Com o aumento de sua utilização ocorre a preocupação para a sua produção. A energia fotovoltaica tem se destacado por ser limpa, constante e sem alteração ao meio ambiente com sua instalação, aliado aos altos indices de irradiação solar no Brasil. Nesse trabalho foi feito um estudo sobre viabilidade da instalação de um sistema fotovoltaico em um prédio da UFMS. Para tal, foi realizado um levantamento de carga e avaliadas três propostas de sistemas. A primeira atendia somente a iluminação, a segunda proposta era referente a iluminação e computadores, e a terceira atendia a iluminação, os computadores e os condicionadores de ar. Para cada sistema proposto foram definidos a quantidade de painéis, o modelo, e o inversor adequado a ser implantado. Além disso, foi estudada a viabilidade da utilização de estrutura de elevação. Por se tratar de um investimento que tem vida útil média de 25 anos, foi realizada a análise financeira do período, através do payback simples, o Valor Presente Líquido (VPL) e a Taxa Interna de Retorno (TIR), afim de se verificar a viabilidade no investimento em sistema fotovoltaico.

Palavras-chave - SISTEMA, SOLAR, FOTOVOLTAICO, VIABILIDADE ECONÔMICA.

\section{TECHNICAL AND ECONOMIC FEASIBILITY ANALYSIS OF LIFTING STRUCTURE FOR A PHOTOVOLTAIC SYSTEM IN A UNIVERSITY BUILDING}

Electricity has become increasingly important in society over the years. With the increase of its use, the concern for its production occurs. Photovoltaic energy has been outstanding for being clean, constant and without alteration to the environment with its installation, allied to the high rates of solar irradiation in Brazil. In this work a study was made about the feasibility of installing a photovoltaic system in a UFMS building. For this, a load survey was carried out and three system proposals were evaluated. The first was for lighting only, the second was for lighting and computers, and the third was for lighting, computers, and air conditioners. For each proposed system, the number of panels, the model, and the appropriate inverter to be deployed were defined. In addition, the feasibility of using a lifting structure was studied. As it is an investment with an average useful life of 25 years, the financial analysis of the period was performed through the simple payback, the Net Present Value (NPV) and the Internal Rate of Return (IRR), in order to verify the feasibility of investing in photovoltaic system.

Keywords - SYSTEM, SOLAR, PHOVOLTAIC, ECONOMIC FEASIBILITY. 


\section{INTRODUÇÃO}

A matriz energética brasileira tem predominância de fontes hídricas. Entretanto, a dependência da eletricidade gerada por hidroelétricas, que já chegou a quase $82 \%$ de toda a produção nacional no passado (BRASILNOSSO, 2011), tem diminuído, e atualmente o valor é de cerca de 60\% (ANEEL, 2018). Esta redução deve-se principalmente a introdução de outras fontes de energia renovável.

A Internacional Energy Agency (IEA) em 2018 destacou o crescimento de energias renováveis eólica e solar para substituição do antigo cenário elétrico fóssil.

O Brasil apresenta um cenário propício para utilização de energia proveniente do sol, com índice de irradiação entre 1500 a $2400 \mathrm{kWh} \mathrm{m²/ano,} \mathrm{valores} \mathrm{estes} \mathrm{que} \mathrm{na} \mathrm{Alemanha,} \mathrm{vista} \mathrm{como}$ referência mundial em energia fotovoltaica, ficam entre 900 a $1250 \mathrm{kWh} \mathrm{m²/ano} \mathrm{(ASMLatin,} \mathrm{2018).}$

Diante desse cenário propício de produção, há um notável crescimento exponencial do uso de energia solar, que no início de 2017 era de 70\%, quando comparado aos 2 anos anteriores, e com a previsão da matriz energética solar chegar a 10\% do total brasileiro até 2030 (Oglobo, 2017).

A implantação de sistemas fotovoltaicos, que se tem tornado cada vez mais viável, tem como principal objetivo reduzir a conta de energia elétrica, possibilitando que o valor economizado possa ser investido em outro setor da universidade.

Uma vez que a preocupação mundial com relação a fontes de energia renovável tem se ampliado nos últimos anos, afim de evitar um dos assuntos mais problemáticos do século, a poluição, a energia solar tem se destacado, principalmente no Brasil, onde possui cenário propício para este tipo de produção. Isso aliado ao apelo à preservação do meio ambiente, uma vez que a produção de energia fotovoltaica é limpa e não apresenta aspectos negativos à natureza. Nota-se que com a construção de usinas hidrelétricas, que apesar de gerar energia "limpa", apresenta impactos consideráveis ao meio ambiente no momento da sua construção.

A revisão da resolução normativa no 482/2012, que criou o Sistema de Compensação de Energia Elétrica, garante para a produção de energia fotovoltaica mais um aspecto positivo para instalação de um sistema.

Portanto, tem-se neste trabalho, um estudo de viabilidade técnica e econômica da estrutura de elevação para a instalação de um sistema de energia fotovoltaica no ângulo de melhor incidência solar no prédio do curso de Engenharia Elétrica, da Universidade Federal do Mato Grosso do Sul (UFMS).

Diante desse desafio, deve-se fazer uma análise técnica do funcionamento de um sistema fotovoltaico, realizar análise do local escolhido para aplicação do sistema e elaborar os cálculos de dimensionamento do sistema que possam atender iluminação, computadores e ar condicionados, analisando como o investimento em estruturas de elevação impactam na viabilidade do sistema, e por fim, fazer a análise da viabilidade econômica do sistema fotovoltaico para o prédio proposto através do payback simples, taxa interna de retorno (TIR) e valor presente líquido (VPL).

\section{METODOLOGIA}

Para melhor visualização da viabilidade econômica foram analisados três possíveis sistemas:

- $\quad$ Sistema 01 - Atendimento somente da iluminação.

- $\quad$ Sistema 02 - Atendimento de iluminação e computadores.

- Sistema 03 - Atendimento de iluminação, computadores e condicionadores de ar.

O primeiro passo para projetar um sistema fotovoltaico é saber a energia que se deseja produzir.

Como o prédio em questão está dentro do consumidor UFMS, que contém outras edificações (não sendo necessário, então, a retirada da taxa de disponibilidade da energia), isto é, não há acesso a conta de energia exata da edificação em questão, fez-se necessário a realização de 
um levantamento de carga contando a quantidade de lâmpadas, computadores e condicionadores de ar, assim como o tempo médio de uso dos aparelhos.

Após coletar os dados sobre a energia necessária a produzida, e utilizando-se do google maps para encontrar as coordenadas do local e em sequência, utilizando a ferramenta Sundata do site CRESESB, é possível encontrar o índice de irradiação solar no local. Por fim, utilizando a Equação (1), encontra-se a potência de painéis necessárias para geração da energia pretendida.

$$
P_{p}=\frac{E_{d}}{M_{d} x F_{a}}
$$

Onde:

$P_{p}$ - Potência dos painéis [kWp].

$E_{d}$ - Energia diária que precisa se produzir [kWh].

$M_{\text {d }}$ - Média diária de energia do local $\left[\mathrm{kWh} / \mathrm{m}^{2}\right.$. dia].

$F_{a}$ - Fator de ajuste.

Com a potência e o modelo de painéis escolhido, é possível calcular a quantidade de placas fotovoltaicas necessárias através da Equação (2).

$$
N_{p}=\frac{1000 \times P_{p}}{P_{i}}
$$

Onde:

$N_{p}$ - Número de painéis.

$P_{p-}$ Potência dos painéis [kWp].

$P_{i-}$ Potência de pico individual do painel [Wp].

Por fim, para seleção do inversor é necessário utilizar as Equações (3) e (4), em conjunto com a análise do datasheet dos modelos pretendidos, afim de encontrar o melhor arranjo de painéis possíveis entre as MPPT do inversor, cuidando com a corrente máxima de entrada no inversor, assim como a corrente de curto circuito produzida pelo painel. Além da corrente, analisase também no datasheet, a tensão máxima de entrada do inversor e a tensão a vazio do painel.

$$
P_{p}=N_{p} x P i
$$

$$
0,8 \times P_{p} \leq P_{\text {inv }} \leq 1,2 \times P_{p}
$$

Onde:

$P_{p}$ - Potência dos painéis [Wp].

$N_{p}$ - Número de painéis que serão utilizados.

$P_{i}$ - Potência individual do painel.

$P_{i n v}$ - Potência do inversor [Wp]. 
Com a definição da quantidade de painéis a serem utilizados em cada Sistema, verificou-se a área de instalação, procurando o norte geográfico, e a diferença que o sistema teria na produção, caso fossem instalados os painéis aproveitando a inclinação do telhado, ou então, com estruturas para que esta inclinação alcance o valor de maior irradiação local.

Com o dimensionamento da quantidade de painéis e dos inversores a serem empregados, foi verificado o projeto que apresentou os melhores resultados, através dos cálculos de viabilidade econômica pelo cálculo de índices de payback simples, VPL e TIR.

\section{RESULTADOS}

No levantamento de carga foi contado cada uma das lâmpadas, computadores e condicionadores de ar, assim como uma média do tempo de uso para todos os cômodos da edificação.

As lâmpadas de $32 \mathrm{~W}$, são fluorescentes tubular, enquanto que as que são $45 \mathrm{~W}$ são fluorescentes espiral, já as poucas que aparecem de 8W são de LED bulbo.

Seguindo a NDU 001 - Energisa, revisada em dez/17, retirado da Tabela 1 da referida norma, foi obtida a potência de computadores como $300 \mathrm{~W}$, e com a Tabela 6 da norma foi obtido o valor de $1400 \mathrm{~W}$ para ar condicionado do tipo janela (10000 BTUs).

O levantamento de carga completa, com a energia gasta pelos itens e o tempo médio de uso podem ser vistas na Figura 1.

\begin{tabular}{|c|c|c|c|c|c|c|c|c|c|c|c|c|}
\hline \multirow{3}{*}{ Cômodo } & \multicolumn{12}{|c|}{ Cargas } \\
\hline & \multicolumn{4}{|c|}{ Lâmpadas } & \multicolumn{4}{|c|}{ Computador } & \multicolumn{4}{|c|}{ Ar Condicionado } \\
\hline & Número & Potência (W) & Uso (h) & Energia (Wh) & Número & Potência (W) & Uso (h) & Energia (Wh) & Número & Potência (W) & Uso (h) & Energia (Wh) \\
\hline Sala de professor 1 & 4 & 32 & 10 & 1280 & 2 & 300 & 6 & 3600 & 1 & 1400 & 8 & 11200 \\
\hline Sala de professor 2 & 4 & 32 & 10 & 1280 & 2 & 300 & 6 & 3600 & 1 & 1400 & 8 & 11200 \\
\hline Sala de professor 3 & 4 & 32 & 10 & 1280 & 1 & 300 & 8 & 2400 & 1 & 1400 & 8 & 11200 \\
\hline Sala de professor 4 & 4 & 32 & 10 & 1280 & 1 & 300 & 8 & 2400 & 1 & 1400 & 8 & 11200 \\
\hline Sala de professor 5 & 4 & 32 & 10 & 1280 & 2 & 300 & 3 & 1800 & 1 & 1400 & 4 & 5600 \\
\hline Sala de professor 6 & 4 & 32 & 10 & 1280 & 2 & 300 & 6 & 3600 & 1 & 1400 & 8 & 11200 \\
\hline Sala de professor 7 & 4 & 32 & 10 & 1280 & 3 & 300 & 6 & 5400 & 1 & 1400 & 8 & 11200 \\
\hline Mestrado & 8 & 32 & 4 & 1024 & & & & 0 & 2 & 1400 & 4 & 11200 \\
\hline Copa & 1 & 32 & 2 & 64 & & & & 0 & & & & 0 \\
\hline Sala de professor 8 & 4 & 32 & 6 & 768 & 1 & 300 & 6 & 1800 & 1 & 1400 & 6 & 8400 \\
\hline Sala de professor 9 & 2 & 32 & 6 & 384 & 2 & 300 & 6 & 3600 & 1 & 1400 & 8 & 11200 \\
\hline BWC Fem & 4 & 32 & 6 & 768 & & & & 0 & & & & 0 \\
\hline PET & 4 & 32 & 6 & 768 & 1 & 300 & 5 & 1500 & 1 & 1400 & 8 & 11200 \\
\hline BWC Masc & 4 & 32 & 6 & 768 & & & & 0 & & & & 0 \\
\hline Almoxarifado & 1 & 32 & 1 & 32 & & & & 0 & & & & 0 \\
\hline Sala de professor 10 & 1 & 32 & 4 & 128 & 1 & 300 & 4 & 1200 & 1 & 1400 & 4 & 5600 \\
\hline Lab. Embarcados & 2 & 32 & 1 & 64 & & & & 0 & 1 & 1400 & 2 & 2800 \\
\hline Secretaria & 4 & 32 & 8 & 1024 & 1 & 300 & 8 & 2400 & 1 & 1400 & 8 & 11200 \\
\hline Lab. Eletrônica & 18 & 32 & 4 & 2304 & & & & 0 & 1 & 1400 & 4 & 5600 \\
\hline Lab. Eficiência & 18 & 32 & 1 & 576 & & & & 0 & 1 & 1400 & 1 & 1400 \\
\hline Lab. Materiais Elétricos & 18 & 32 & 4 & 2304 & & & & 0 & 1 & 1400 & 4 & 5600 \\
\hline Sala de ténicos & 1 & 32 & 8 & 256 & 1 & 300 & 8 & 2400 & 1 & 1400 & 8 & 11200 \\
\hline Lab. Máquinas & 18 & 32 & 2 & 1152 & & & & 0 & 1 & 1400 & 3 & 4200 \\
\hline Lab. Lamosse & 2 & 32 & 6 & 384 & & & & 0 & 1 & 1400 & 6 & 8400 \\
\hline EngeFour Jr. & 6 & 45 & 10 & 2700 & 6 & 300 & 8 & 14400 & 1 & 1850 & 10 & 18500 \\
\hline Corredor 1 & 3 & 45 & 6 & 810 & & & & & & & & \\
\hline Corredor 2 & 7 & 8 & 6 & 336 & & & & & & & & \\
\hline \multicolumn{4}{|c|}{ TOTAL } & 25574 & & & & 50100 & & & & 189300 \\
\hline
\end{tabular}

Figura 1. Levantamento de carga do prédio de engenharia elétrica. Fonte: Própria (2019). 
Tendo os valores gastos diários, assumindo 21 dias de utilização, a energia mensal a ser produzida para cada um dos sistemas pode ser vista na Figura 2.

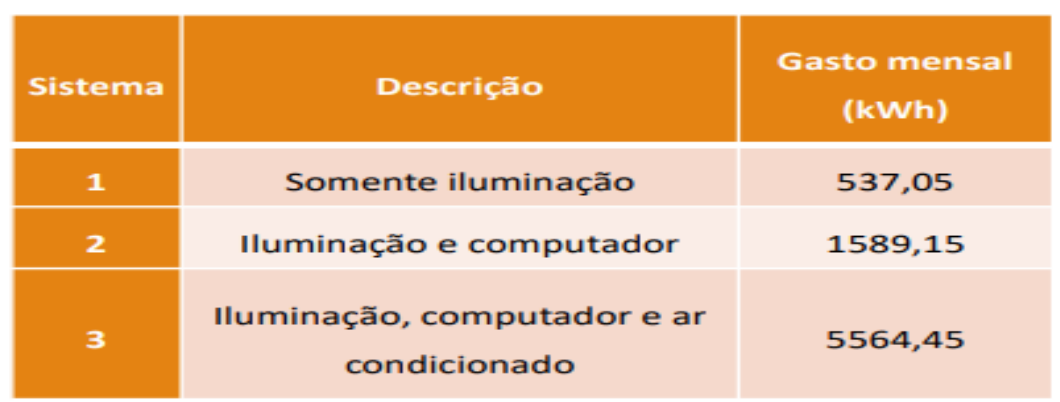

Figura 2. Energia a ser produzida. Fonte: Própria (2019).

\subsection{Quantidade de painéis}

Através do google maps é possível saber as coordenadas geográficas do local, e então utiliza-se a ferramenta Sundata do site CRESESB para encontrar o índice de irradiação, conforme apresentado na Figura 3.

\begin{tabular}{|c|c|c|}
\hline Ângulo & Inclinação & $\begin{array}{c}\text { Irradiação } \\
\text { média anual }\end{array}$ \\
\hline Plano horizontal & $0^{\circ} \mathrm{N}$ & 5,01 \\
\hline Ângulo igual a latitude & $21^{\circ} \mathrm{N}$ & 5,21 \\
\hline Maior média anual & $19^{\circ} \mathrm{N}$ & 5,21 \\
\hline Maior minimo mensal & $26^{\circ} \mathrm{N}$ & 5,18 \\
\hline
\end{tabular}

Figura 3. Dados de irradiação. Fonte: CRESESB adaptada (2019).

Com o dado de irradiação do local e assumindo um fator de ajuste 0,8, para correção da perda de potência na produção, por efeito joule nos cabos, no próprio inversor, perdas por temperatura, incompatibilidade elétrica e acúmulo de sujeira, é encontrada a potência de painéis necessária para geração da energia requisitada através da Equação (1) e os resultados podem ser vistos na Figura 4. Para as duas situações propostas tem-se diferentes valores de irradiação, levando em consideração o plano horizontal onde a irradiação solar diária média anual é de 5,01 $\mathrm{kWh} / \mathrm{m}^{2}$.dia e o ângulo de maior média anual de incidência (19ㅇ) possui irradiação de 5,21 $\mathrm{kWh} / \mathrm{m}^{2}$.dia.

\begin{tabular}{|c|c|c|}
\hline & \multicolumn{2}{|c|}{ Potência dos painéis (kWp) } \\
\hline Sistema & $\begin{array}{c}\text { Plano horizontal (sem } \\
\text { estrutura de elevação) }\end{array}$ & $\begin{array}{c}\text { Inclinação de } 19^{\circ} \text { (com } \\
\text { estrutura de elevação) }\end{array}$ \\
\hline 1 & 4,47 & 4,30 \\
\hline 2 & 13,22 & 12,71 \\
\hline 3 & 46,28 & 44,50 \\
\hline
\end{tabular}

Figura 4. Potência de painéis. Fonte: Própria (2019).

Com a potência e o modelo dos painéis, é possível calcular a quantidade de painéis utilizando a Equação (2), como pode ser visto na Figura 5. O painel a ser utilizado é o Canadian 330 Wp. 


\begin{tabular}{|c|c|c|}
\hline & \multicolumn{2}{|c|}{ Quantidade de painéis } \\
\hline Sistema & $\begin{array}{c}\text { Plano horizontal (sem } \\
\text { estrutura de elevação) }\end{array}$ & $\begin{array}{c}\text { Inclinação de } 19^{\circ} \text { (com } \\
\text { estrutura de elevação) }\end{array}$ \\
\hline 1 & 13,5 & 13,0 \\
\hline 2 & 40,1 & 38,5 \\
\hline 3 & 140,2 & 134,9 \\
\hline
\end{tabular}

Figura 5. Quantidade de painéis. Fonte: Própria (2019).

\subsection{Viabilidade em sistema de elevação}

Se for considerado a inclinação ideal, para o Sistema 3, são necessários 5 painéis a menos quando comparado com o plano horizontal (telhado de fibrocimento da edificação com angulação mínima) para produção da mesma quantidade de energia enquanto que para o menor sistema (Sistema 1), que atende somente a potência de iluminação, e possui gasto mensal em torno de 537 kWh a diferença é de menos de um painel. Para o sistema 2, a diferença é de dois painéis.

Tendo preços analisados em duas empresas, valores atualizados para agosto de 2019, para a empresa A o valor unitário do painel é de $\mathrm{R} \$ 719,00$ e o kit de montagem para 4 painéis para elevação é de $R \$ 859,00$. Para a empresa $B$, o painel custa $R \$ 631,47$ enquanto o kit para elevação de 4 painéis, custa $\mathrm{R} \$ 1.208,07$, conforme o resumo contido na Figura 6 . O comparativo dos preços dos sistemas pode ser visto na Figura 7.

\begin{tabular}{|l|c|c|}
\hline & Preço painel & Preço kit \\
\hline Empresa A & $\mathrm{R} \$ 719,00$ & $\mathrm{R} \$ 859,00$ \\
\hline Empresa B & $\mathrm{R} \$ 631,47$ & $\mathrm{R} \$ 1.208,07$ \\
\hline
\end{tabular}

Figura 6. Resumo dos preços de painel e kit de elevação. Fonte: Própria (2019).

\begin{tabular}{|c|c|c|c|c|c|c|c|c|c|}
\hline & \multirow{2}{*}{$\begin{array}{c}\text { Quantidade } \\
\text { paineis }\end{array}$} & \multirow{2}{*}{$\begin{array}{c}\text { Kits de } 4 \\
\text { paineis }\end{array}$} & \multicolumn{3}{|c|}{ Empresa A } & \multicolumn{3}{|c|}{ Empresa B } \\
\hline & & & & Preço painéis & Preço kit & Preço total & Preço painéis & Preço kit & Preço total \\
\hline \multirow{2}{*}{ Sistema 1} & SEM elevação & 13 & 0 & $\mathrm{R} \$ 9.347,00$ & $\mathrm{RSO}, 00$ & RS9.347,00 & R\$8.209,11 & $\mathrm{RSO}, 00$ & RSS.209,11 \\
\hline & COM elevação & 13 & 4 & $\mathrm{R} \$ 9.347,00$ & R\$3.436,00 & RS12.783,00 & R\$8.209,11 & $\mathrm{R} \$ 4.832,28$ & R\$13.041,39 \\
\hline \multirow{2}{*}{ Sistema 2} & SEM elevação & 40 & 0 & R\$28.760,00 & RSO,00 & R\$28.760,00 & $\mathrm{R} \$ 25.258,80$ & RSO 000 & 8,80 \\
\hline & COM elevação & 38 & 10 & R\$27.322,00 & R\$8.590,00 & R\$35.912,00 & R\$23.995,86 & RS12.080,70 & RS36.076,56 \\
\hline \multirow{2}{*}{ Sistema 3} & SEM elevação & 140 & 0 & $\mathrm{R} \$ 100.660,00$ & $\mathrm{RS} 0,00$ & $\mathrm{R} \$ 100.660,00$ & R\$88.405,80 & $\mathrm{RSO}_{2}, 00$ & R\$s8.405,80 \\
\hline & COM elevação & 135 & 34 & R\$97.065,00 & R\$29.206,00 & R\$126.271,00 & R\$85.248,45 & RS41.074,38 & RS126.322,83 \\
\hline
\end{tabular}

Figura 7. Comparativo da viabilidade da estrutura de elevação. Fonte: Própria (2019).

Pelo comparativo é possível analisar que mesmo necessitando de mais painéis, os sistemas que acompanham a inclinação do telhado são mais viáveis economicamente, e que para sistemas menores, a diferença de produção é mínima, a ponto da utilização de sistemas de elevação não refletir nem ao menos na diminuição de um painel.

\subsection{Seleção dos inversores}

A potência ideal para o inversor é relacionada com a potência de painéis colocada uma margem de $20 \%$ para mais e menos, então usa-se nesta etapa as Equações (3) e (4). O ideal é trabalhar dentro do range para menos, uma vez que a potência do painel é $W p$, então é esperável que nem sempre atinja este valor de pico. Como o Sistema 3 é consideravelmente grande, foi dividido em dois sistemas, 3.1 e 3.2, exatamente iguais. O range dos inversores para os três sistemas podem ser vistos na Figura 8. 


\begin{tabular}{|c|c|c|}
\hline Sistema & $\begin{array}{c}\text { Potência } \\
\text { painéis }(\mathrm{Wp})\end{array}$ & Range inversor \\
\hline 1 & 4290 & $3432 \leq \mathrm{P}$ inv $\geq 5148$ \\
\hline 2 & 13200 & $10560 \leq \mathrm{P}$ inv $\geq 15840$ \\
\hline 3.1 e 3.2 & 23100 & $18480 \leq \mathrm{P}$ inv $\geq 27720$ \\
\hline
\end{tabular}

Figura 8. Range do inversor. Fonte: Própria (2019).

Outro fator decisivo na seleção dos inversores são as correntes e tensões máximas suportadas pelas MPPT do inversor. Nessa etapa é importante a adequação do arranjo de painéis afim de conseguir uma configuração suportada pelo inversor, uma vez que quando painéis são colocados em série, a tensão deles é somada, e a cada paralelo (chamado string) feito de painéis em série, o que se soma é a corrente.

Importante notar que para o Sistema 2, que demanda 40 painéis, é comum pensar em dividir o sistema igualmente entre as MPPT, mas é necessário analisar o datasheet do inversor, porque normalmente a MPPT 1 acaba suportando mais corrente, portanto, o melhor arranjo pode ser visto na Figura 9, assim como o modelo do inversor.

\begin{tabular}{|c|c|c|c|c|}
\hline & inéis & MPPT 1 & MPF & or \\
\hline \multirow{2}{*}{ Sistema 1} & & 13 séries & & \multirow{2}{*}{ Fronius Primo $4.0-1$} \\
\hline & & 1 string & & \\
\hline \multirow{2}{*}{ Sistema 2} & \multirow{2}{*}{0} & 15 séries & 10 séries & \multirow{2}{*}{ Fronius Symo 12.5-3-M } \\
\hline & & 2 strings & 1 string & \\
\hline \multirow{2}{*}{ Sistema } & \multirow{2}{*}{70} & 18 séries & 17 séries & \multirow{2}{*}{ Fronius Symo 20.0-3-M } \\
\hline & & 2 strings & 2 strings & \\
\hline
\end{tabular}

Figura 9. Arranjo e modelo do inversor. Fonte: Própria (2019).

\subsection{Levantamento de preço}

Uma ideia inicial pode ser adquirida ao comparar preços dos equipamentos selecionados em diversos sites, mas somente uma proposta da empresa transmitirá de fato o valor real, porque nesse orçamento completo, serão acrescentados outros componentes como cabos, disjuntores, fusíveis, DPS e a mão de obra. O orçamento total dado por uma empresa, para os três sistemas propostos pode ser visto na Figura 10.

\begin{tabular}{|c|c|}
\hline \multicolumn{2}{|c|}{ Preço orçado final } \\
\hline Sistema 1 & $\mathrm{R} \$ 28.995,80$ \\
\hline Sistema 2 & $\mathrm{R} \$ \mathbf{7 4 . 9 6 4 , 8 4}$ \\
\hline Sistema 3 & $\mathrm{R} \$ 208.445,54$ \\
\hline
\end{tabular}

Figura 10. Levantamento de preço dos sistemas. Fonte: Própria (2019).

\subsection{Vida útil dos equipamentos e preço da energia}

A expectativa de vida útil e durabilidade dos painéis, em média, é de 25 anos, onde os fabricantes garantem $90 \%$ da potência até o décimo segundo ano (considerando a perda linear, seriam $0,83 \%$ de perda anual nos 12 primeiros anos) e 80\% em 25 anos (0,77\% nos 13 últimos anos). (SolarVolt, 2015) 
Os inversores, segundo Ecycle, possuem vida útil variando entre 10 a 15 anos, portanto neste trabalho, para o inversor, foi assumida uma vida útil de 10 anos.

Já em relação ao preço da energia, no ano de 2015, houveram três elevações no preço (PROGRESSO, 2015). Em 2016, a ANEEL aprovou aumento de 7,4\% na conta de energia para a Energisa Mato Grosso do Sul $(G 1,2016)$. Diante de um histórico de aumento de energia, para este trabalho então foi considerado um aumento anual, para os próximos 25 anos, de $5 \%$ no preço da energia, tendo como valor inicial $\mathrm{R} \$ 0,83$ por $\mathrm{kWh}$, que exprime uma média do valor da energia e seus encargos para abril de 2019.

\subsection{Cálculo da viabilidade econômica}

Com os itens e o valor dos sistemas é possível calcular a viabilidade econômica do sistema, começando no ano 0 com valor negativo relativo ao custo inicial de implantação e nos anos 10 e 20 o valor negativo relacionado com a troca de inversor. Os cálculos ano a ano, para os três sistemas propostos, podem ser analisados nas Figuras 11, 12 e 13.

\begin{tabular}{|c|c|c|c|c|}
\hline \multicolumn{5}{|c|}{ Sistema 1 } \\
\hline Ano & Produção anual $(\mathrm{kWh})$ & Preço do $\mathrm{kWh}$ & Fluxo de caixa & Saldo \\
\hline 0 & \multicolumn{2}{|c|}{ Custo inicial do sistema } & R $\$ 28.995,80$ & -R $28.995,80$ \\
\hline 1 & 6189,96 & $\mathrm{R} \$ 0,83$ & $\mathrm{R} \$ 5.137,67$ & -R $\$ 23.858,13$ \\
\hline 2 & 6138,58 & $\mathrm{R} \$ 0,87$ & $\mathrm{R} \$ 5.349,78$ & -R $\$ 18.508,36$ \\
\hline 3 & 6087,63 & $\mathrm{R} \$ 0,92$ & $\mathrm{R} \$ 5.570,64$ & -R $\$ 12.937,72$ \\
\hline 4 & 6037,11 & $\mathrm{R} \$ 0,96$ & $\mathrm{R} \$ 5.800,62$ & -R $\$ 7.137,09$ \\
\hline 5 & 5987,00 & $\mathrm{R} \$ 1,01$ & $\mathrm{R} \$ 6.040,10$ & -R $\$ 1.096,99$ \\
\hline 6 & 5937,31 & $\mathrm{R} \$ 1,06$ & $\mathrm{R} \$ 6.289,47$ & $\mathrm{R} \$ 5.192,48$ \\
\hline 7 & 5888,03 & $\mathrm{R} \$ 1,11$ & $\mathrm{R} \$ 6.549,13$ & $\mathrm{R} \$ 11.741,61$ \\
\hline 8 & 5839,16 & $\mathrm{R} \$ 1,17$ & $\mathrm{R} \$ 6.819,51$ & $\mathrm{R} \$ 18.561,12$ \\
\hline 9 & 5790,69 & $\mathrm{R} \$ 1,23$ & $\mathrm{R} \$ 7.101,05$ & $\mathrm{R} \$ 25.662,18$ \\
\hline \multirow{2}{*}{10} & 5742,63 & $\mathrm{R} \$ 1,29$ & $\mathrm{R} \$ 7.394,22$ & $\mathrm{R} \$ 33.056,40$ \\
\cline { 2 - 6 } & \multicolumn{2}{|c|}{$\mathrm{Troca}$ de inversor } & $-\mathrm{R} \$ 9.590,00$ & $\mathrm{R} \$ 23.466,40$ \\
\hline 11 & 5694,96 & $\mathrm{R} \$ 1,35$ & $\mathrm{R} \$ 7.699,49$ & $\mathrm{R} \$ 31.165,89$ \\
\hline 12 & 5647,70 & $\mathrm{R} \$ 1,42$ & $\mathrm{R} \$ 8.017,37$ & $\mathrm{R} \$ 39.183,25$ \\
\hline 13 & 5604,21 & $\mathrm{R} \$ 1,49$ & $\mathrm{R} \$ 8.353,41$ & $\mathrm{R} \$ 47.536,67$ \\
\hline 14 & 5561,06 & $\mathrm{R} \$ 1,57$ & $\mathrm{R} \$ 8.703,55$ & $\mathrm{R} \$ 56.240,21$ \\
\hline 15 & 5518,24 & $\mathrm{R} \$ 1,64$ & $\mathrm{R} \$ 9.068,36$ & $\mathrm{R} \$ 65.308,57$ \\
\hline 16 & 5475,75 & $\mathrm{R} \$ 1,73$ & $\mathrm{R} \$ 9.448,46$ & $\mathrm{R} \$ 74.757,02$ \\
\hline 17 & 5433,58 & $\mathrm{R} \$ 1,81$ & $\mathrm{R} \$ 9.844,49$ & $\mathrm{R} \$ 84.601,51$ \\
\hline 18 & 5391,74 & $\mathrm{R} \$ 1,90$ & $\mathrm{R} \$ 10.257,12$ & $\mathrm{R} \$ 94.858,63$ \\
\hline 19 & 5350,23 & $\mathrm{R} \$ 2,00$ & $\mathrm{R} \$ 10.687,05$ & $\mathrm{R} \$ 105.545,68$ \\
\hline \multirow{2}{*}{20} & 5309,03 & $\mathrm{R} \$ 2,10$ & $\mathrm{R} \$ 11.134,99$ & $\mathrm{R} \$ 116.680,67$ \\
\cline { 2 - 6 } & \multicolumn{2}{|c|}{ Troca de inversor } & $-\mathrm{R} \$ 9.590,00$ & $\mathrm{R} \$ 107.090,67$ \\
\hline 21 & 5268,15 & $\mathrm{R} \$ 2,20$ & $\mathrm{R} \$ 11.601,72$ & $\mathrm{R} \$ 118.692,39$ \\
\hline 22 & 5227,59 & $\mathrm{R} \$ 2,31$ & $\mathrm{R} \$ 12.088,00$ & $\mathrm{R} \$ 130.780,39$ \\
\hline 23 & 5187,33 & $\mathrm{R} \$ 2,43$ & $\mathrm{R} \$ 12.594,67$ & $\mathrm{R} \$ 143.375,07$ \\
\hline 24 & 5147,39 & $\mathrm{R} \$ 2,55$ & $\mathrm{R} \$ 13.122,58$ & $\mathrm{R} \$ 156.497,64$ \\
\hline 25 & 5107,76 & $\mathrm{R} \$ 2,68$ & $\mathrm{R} \$ 13.672,61$ & $\mathrm{R} \$ 170.170,25$ \\
\hline
\end{tabular}

Figura 11. Análise do Sistema 1. Fonte: Própria (2019). 


\begin{tabular}{|c|c|c|c|c|}
\hline \multicolumn{5}{|c|}{ Sistema 2} \\
\hline Ano & Produção anual (kWh) & Preço do $\mathrm{kWh}$ & Fluxo de caixa & Saldo \\
\hline 0 & \multicolumn{2}{|c|}{ Custo inicial do sistema } & -R\$74.964,84 & -R\$74.964,84 \\
\hline 1 & 19046,02 & RS0,83 & R\$15.808,20 & -R\$59.156,64 \\
\hline 2 & 18887,94 & $\mathrm{RS} 0,87$ & R\$16.460,84 & -R\$42.695,81 \\
\hline 3 & & & R\$17.1 & -R\$25.555,38 \\
\hline 4 & & & R\$17 & -R\$7.707,32 \\
\hline 5 & 18421,52 & 01 & RS18 & R\$10.877,61 \\
\hline 6 & 62 & & $\mathrm{R} \$ 1$ & $\mathrm{R} \$ 30.229,81$ \\
\hline 7 & & & R\$2 & $\mathrm{R} \$ 50.38$ \\
\hline 8 & & & R\$2 & R\$71.3 \\
\hline 9 & 17817,50 & & R\$21 & R\$93.213,45 \\
\hline \multirow{2}{*}{10} & 17669,61 & $\mathrm{RS}$ & R\$22.751,44 & R\$115.964,89 \\
\hline & \multicolumn{2}{|c|}{ Troca de inversor } & $-\mathrm{R} \$ 9.590,00$ & R\$106.374,89 \\
\hline 11 & 17522,96 & $R \$ 1,35$ & R\$23.690,73 & R\$130.065,62 \\
\hline 12 & 1737 & $\mathrm{RS}$ & $R \$ 24.668,80$ & $\mathrm{R} \$ 154.734,42$ \\
\hline 13 & 71 & $\overline{R S}$ & R\$25.702,80 & R\$180.437,22 \\
\hline 14 & & & R\$26 & R\$207.217,34 \\
\hline 15 & & & R\$2 & R\$235.119,96 \\
\hline 16 & & & $\mathrm{RS} 2 \mathrm{C}$ & $\mathrm{R} \$ 264.192,12$ \\
\hline 17 & & & R\$3 & R\$294.482,83 \\
\hline 18 & & & $\mathrm{RS} 3$ & $\mathrm{R} \$ 326$. \\
\hline 19 & & & $\mathrm{R} \$ 3$ & $\mathrm{R} \$ 358.926,38$ \\
\hline \multirow{2}{*}{20} & 16335,47 & R\$2,10 & R\$34.261,50 & R\$393.187,88 \\
\hline & \multicolumn{2}{|c|}{ Troca de inversor } & $-R \$ 9.590,00$ & R\$383.597,88 \\
\hline 21 & 16209,69 & $R \$ 2,20$ & $R \$ 35.697,57$ & R\$419.295,46 \\
\hline 22 & 16084,87 & $\mathrm{R} \$ 2,31$ & $\mathrm{R} \$ 37.193,84$ & R\$456.489,29 \\
\hline 23 & 15961,02 & $R \$ 2,43$ & $\mathrm{R} \$ 38.752,82$ & R\$495.242,11 \\
\hline 24 & 1583 & $R \$ 2,55$ & $\mathrm{R} \$ 40.377,14$ & R\$535.619,25 \\
\hline 25 & 15716,16 & $R \$ 2,68$ & R\$42.069,55 & $\mathrm{R} \$ 577.688,79$ \\
\hline
\end{tabular}

Figura 12. Análise do Sistema 2. Fonte: Própria (2019).

\begin{tabular}{|c|c|c|c|c|}
\hline \multicolumn{5}{|c|}{ Sistema 3} \\
\hline Ano & Produção anual (kWh) & Preço do kWh & Fluxo de caixa & Saldo \\
\hline 0 & \multicolumn{2}{|c|}{ Custo inicial do sistema } & -R\$208.455,54 & -R\$208.455,54 \\
\hline 1 & 66661,06 & RSO,83 & R\$55.328,68 & $-\mathrm{R} \$ 153.126,86$ \\
\hline 2 & 66107,77 & RSO,87 & RS57.612,92 & -R\$95.513,94 \\
\hline 3 & 65559,08 & RSO,92 & R\$59.991,47 & $-\mathrm{R} \$ 35.522,46$ \\
\hline 4 & 65014,94 & RSO,96 & R\$62.468,22 & $\mathrm{R} \$ 26.945,76$ \\
\hline 5 & 64475,31 & $\mathrm{R} \$ 1,01$ & $\mathrm{R} \$ 65.047,22$ & R\$91.992,98 \\
\hline 6 & 63940,17 & $\mathrm{R} \$ 1,06$ & R\$67.732,70 & RS159.725,68 \\
\hline 7 & 63409,47 & $\mathrm{R} \$ 1,11$ & R\$70.529,04 & $\mathrm{R} \$ 230.254,72$ \\
\hline 8 & 62883,17 & R\$1,17 & R\$73.440,83 & $\mathrm{R} \$ 303.695,55$ \\
\hline 9 & 62361,24 & $\mathrm{R} \$ 1,23$ & R\$76.472,84 & $\mathrm{R} \$ 380.168,39$ \\
\hline \multirow{2}{*}{10} & 61843,64 & R\$1,29 & R\$79.630,02 & R\$459.798,41 \\
\hline & \multicolumn{2}{|c|}{ Troca de inversor } & -R\$9.590,00 & R\$450.208,41 \\
\hline 11 & 61330,34 & $\mathrm{R} \$ 1,35$ & RS82.917,54 & RS533.125,95 \\
\hline 12 & 60821,29 & $\mathrm{R} \$ 1,42$ & RS86.340,79 & RS619.466,75 \\
\hline \begin{tabular}{|l|}
13 \\
\end{tabular} & 60352,97 & $\mathrm{R} \$ 1,49$ & R\$89.959,77 & R\$709.426,52 \\
\hline \begin{tabular}{|l|}
14 \\
\end{tabular} & 59888,25 & $\mathrm{R} \$ 1,57$ & R\$93.730,43 & RS803.156,95 \\
\hline \begin{tabular}{|l|}
15 \\
\end{tabular} & 59427,11 & $\mathrm{R} \$ 1,64$ & RS97.659,14 & RS900.816,10 \\
\hline \begin{tabular}{|l|}
16 \\
\end{tabular} & 58969,52 & $\mathrm{R} \$ 1,73$ & $R \$ 101.752,53$ & RS1.002.568,62 \\
\hline \begin{tabular}{|l|}
17 \\
\end{tabular} & 58515,46 & $\mathrm{R} \$ 1,81$ & R\$106.017,48 & R\$1.108.586,11 \\
\hline 18 & 58064,89 & $\mathrm{R} \$ 1,90$ & R\$110.461,21 & R\$1.219.047,32 \\
\hline \begin{tabular}{|l|}
19 \\
\end{tabular} & 57617,79 & $\mathrm{R} \$ 2,00$ & RS115.091,19 & RS1.334.138,50 \\
\hline \multirow[b]{2}{*}{20} & 57174,13 & R\$2,10 & RS119.915,24 & RS1.454.053,74 \\
\hline & \multicolumn{2}{|c|}{ Troca de inversor } & -R\$9.590,00 & RS1.444.463,74 \\
\hline \begin{tabular}{|l|}
21 \\
\end{tabular} & 56733,89 & $\mathrm{R} \$ 2,20$ & R\$124.941,48 & R\$1.569.405,22 \\
\hline \begin{tabular}{|l|}
22 \\
\end{tabular} & 56297,04 & $R \$ 2,31$ & R\$130.178,41 & RS1.699.583,63 \\
\hline \begin{tabular}{|l|}
23 \\
\end{tabular} & 55863,55 & $R \$ 2,43$ & \begin{tabular}{|l|}
$R \$ 135.634,83$ \\
\end{tabular} & R\$1.835.218,46 \\
\hline 24 & 55433,41 & $R \$ 2,55$ & R\$141.319,97 & RS1.976.538,43 \\
\hline \begin{tabular}{|l|}
25 \\
\end{tabular} & 55006,57 & $R \$ 2,68$ & R\$147.243,39 & RS2.123.781,82 \\
\hline
\end{tabular}

Figura 13. Análise do Sistema 3. Fonte: Própria (2019). 
Como taxa média de atratividade, foi considerada a Selic de 6,4\% ao ano, referente ao dia $30 / 04 / 2019$, uma vez que a poupança rende apenas $70 \%$ deste valor ( $4,48 \%$ ao ano), enquanto a Selic estiver abaixo de 8,5\%. Para o cálculo da TIR e VPL, foi utilizada a própria ferramenta do Excel. Um resumo do investimento após 25 anos pode ser visto na Figura 14.

\begin{tabular}{|l|cr|c|c|c|}
\hline & \multicolumn{1}{|c|}{ Investimento inicial } & Payback & TIR & VPL Selic \\
\hline Sistema 1 & RS & $28.995,80$ & 6 anos & $20 \%$ & R\$54.291,20 \\
\hline Sistema 2 & RS & $74.964,84$ & 5 anos & $24 \%$ & R $\$ 196.456,98$ \\
\hline Sistema 3 & RS & $208.455,54$ & 4 anos & $30 \%$ & R\$759.761,96 \\
\hline
\end{tabular}

Figura 14. Análise do investimento. Fonte: Própria (2019)

\section{CONCLUSÃO}

Este artigo apresentou o dimensionamento de placas e inversores de modo a atenderem os 3 tipos de sistemas propostos, assim como o arranjo que melhor se encaixa. Tendo para o Sistema 1, 13 painéis com o inversor Fronius Primo 4.0-1. O Sistema 2, composto de 40 painéis e o inversor Fronius Symo 12.5-3-M. Já o Sistema 3 apresenta 140 painéis com dois inversores Fronius Symo 20.0-3-M.

Foi feita a viabilidade da utilização de estruturas para elevação dos painéis do ângulo de 50, do telhado de fibrocimento do prédio em questão, para o ângulo de 19으, que apresentava a melhor média mensal de irradiação, chegando-se a conclusão que não é viável para nenhuma das empresas selecionadas em nenhum dos sistemas propostos.

A verificação da viabilidade econômica dos sistemas foi realizada através de três índices econômicos diferentes, pelo payback simples, VPL e pela TIR.

A TIR mostra um percentual de retorno do investimento nos sistemas, que quando superior as taxas mínimas de atratividade resultam em um investimento viável.

Já o VPL vai um pouco além, uma vez que ele se refere ao valor que se terá no futuro a mais caso seja optado pelo investimento no sistema fotovoltaico, quando comparado com um investimento que rende a taxa Selic (deixar o dinheiro na poupança, nos dias de hoje, significaria render apenas $70 \%$ dessa taxa Selic).

O resultado obtido para o Sistema 1, com um investimento inicial de $\mathrm{R} \$ \mathbf{2 8 . 9 9 5 , 8 0}$ foi um payback simples de 6 anos, com uma taxa interna de retorno de $20 \%$ e um VPL, quando comparado a Selic, de R\$54.291,20. Para o Sistema 2, que possui investimento inicial de $\mathrm{R} \$ 74.964,84,0$ payback simples foi de 5 anos, com uma TIR de 24\% e um VPL de R\$ 196.456,98. Já o Sistema 3, que apresenta investimento inicial de $\mathrm{R} \$ \mathbf{2 0 8 . 4 5 5 , 5 4}$, teve tempo de payback simples em 4 anos, com uma TIR de 30\% e um VPL de R\$759.761,96.

Portanto, comprova-se que todos os três sistemas propostos se mostram viáveis por possuírem TIR maior que a taxa de atratividade e um VPL positivo. Sendo o Sistema 3, composto por lâmpadas, computadores e condicionador de ar, o sistema que mais se mostrou rentável. 


\section{REFERÊNCIAS BIBLIOGRÁFICAS}

[1] ANEEL. Matriz de Energia Elétrica. 2018. Disponível em: <http://www2.aneel.gov.br/aplicacoes/capacidadebrasil/OperacaoCapacidadeBrasil.cfm>. Acesso em: 11 abr. 2018.

[2] ANEEL. RESOLUÇÃO NORMATIVA № 482. RESOLUÇÃO NORMATIVA № 482, DE 17 DE ABRIL DE 2012, [S.L], 17./jun. 2018. Disponível em: <http://www2.aneel.gov.br/cedoc/ren2012482.pdf>. Acesso em: 08 mai. 2018.

[3] ASMLATIN, Equipe de Conteúdo ASM. Energia elétrica no Brasil em 2018: energia eólica e fotovoltaica. Disponível em: <http://www.asmlatin.com/2018/02/energia-eletrica-no-brasil-em2018-energia-eolica/>. Acesso em: 10 maio 2018.

[4] IEA, International Energy Agency. The Year of Electricity at the IEA. 2018. Disponivel em: <https://www.iea.org/newsroom/news/2018/april/the-year-of-electricity-at-the-iea.html>. Acesso em: 10 maio 2018.

[5] G1. Aneel aprova aumento de 10,75\% na conta de energia elétrica residencial em ms. Disponível

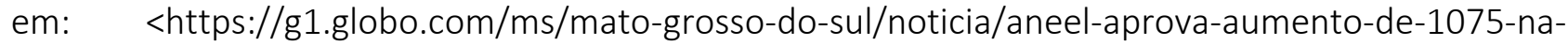
conta-de-energia-eletrica-residencial-em-ms.ghtml>. Acesso em: 25 mai. 2018.

[6] OGLOBO. Energia solar cresceu $70 \%$ em dois anos. Disponível em: <https://oglobo.globo.com/economia/energia-solar-cresceu-70-em-dois-anos-20715504>. Acesso em: 12 mai. 2018.

[7] PROGRESSO. Tarifa de energia sobe pela 3a vez em ms. Disponível em: <http://www.progresso.com.br/dia-a-dia/tarifa-de-energia-sobe-pela-3-vez-em-ms>. Acesso em: 06 jun. 2018.

[8] SOLARVOLTENERGIA. Kit de energia solar: qual a vida útil dos equipamentos? Disponível em: <http://www.solarvoltenergia.com.br/kit-de-energia-solar-vida-util/>. Acesso em: 08 mar. 2018. 